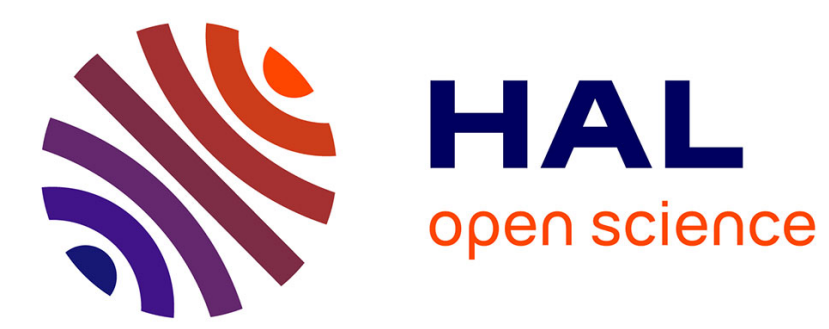

\title{
La forêt paysanne dans les Pyrénées centrales
}

Jean-Paul Métailié

\section{To cite this version:}

Jean-Paul Métailié. La forêt paysanne dans les Pyrénées centrales. Revue Géographique des Pyrénées et du Sud-Ouest, 1984, 55 (2), pp.231 - 238. hal-01496467

\section{HAL Id: hal-01496467 \\ https://hal-univ-tlse2.archives-ouvertes.fr/hal-01496467}

Submitted on 27 Mar 2017

HAL is a multi-disciplinary open access archive for the deposit and dissemination of scientific research documents, whether they are published or not. The documents may come from teaching and research institutions in France or abroad, or from public or private research centers.
L'archive ouverte pluridisciplinaire HAL, est destinée au dépôt et à la diffusion de documents scientifiques de niveau recherche, publiés ou non, émanant des établissements d'enseignement et de recherche français ou étrangers, des laboratoires publics ou privés. 
REVUE GÉOGAPHIQUE DES PYRÉNÉES ET DU SUD-OUEST TOME 55, FASC. 2, pp. 231-238, Toulouse, 1984.

\title{
La forêt paysanne dans les Pyrénées centrales
}

\author{
par Jean.Paul Métailié *
}

Les forêts des Pyrénées centrales ont subi depuis des millénaires des interventions humaines multiples et continues. Les premiers défrichements attestés par la palynologie remontent à une période comprise entre 5000 et 8000 BP sur le piémont voisin de Lourdes. Ils sont plus tardifs et apparaissent vers $3500-4000$ BP dans les vallées internes. On a donc dès le néolithique une modification de la végétation sur un fond de milieux très hétérogènes et très contrastés du point de vue topographique aussi bien que climatique ou géomorphologique. L'effet de l'activité humaine a été très inégal selon les situations et on a aujourd'hui une mosaïque complexe, reflet de la combinaison des contraintes naturelles et du poids des gestions passées et présentes de l'espace. Des schémas d'organisation classique de l'espace sont bien reconnaissables : étagement des formations végétales, phénomènes d'abri, oppositions de versants, etc. Mais les aberrations par rapport à ces schémas abondent. Ces entorses aux modèles naturels sont importantes, car généralement révélatrices des relations entre la société et son environnement végétal. La forêt se révèle comme un lieu privilégié pour l'étude de ces aberrations, parce qu'elle est à la fois profondément humanisée, enjeu économique fondamental et endroit beaucoup plus " sauvage » que les pâturages. Outre les sources habituelles (archives forestières et communales, descriptions naturalistes anciennes...), notre recherche est fondée sur les photos de paysages du $\mathrm{XIX}^{\circ}$ siècle; elles permettent d'avoir un concentré d'informations difficiles à obtenir par les archives habituelles, par exemple sur la physionomie des peuplements. Ces sources, parfois très abondantes dans certaines vallées, peuvent déboucher sur de véritables monographies d'histoire des paysages et une photothèque pyrénéenne est en cours de constitution.

*Attaché de recherches au CNRS, CIMA, LA 366, Université de ToulouseLe Mirail. 
L'organisation de l'espace forestier dans les Pyrénées est dominée par deux faits principaux.

D'une part, la forêt a toujours été considérée par les montagnards comme un espace pastoral à part entière. Le but recherché était la production de nourriture pour le bétail au sens large : herbe, émonde, glands, etc. Cette organisation a donc privilégié, directement ou d'une façon induite, un certain type de physionomie forestière et une certaine composition floristique en fonction des potentialités biologiques et des nécessités économiques. Certains boisements de soulanes et de bas de versants, qui furent très tôt indispensables pour les demisaisons, ont été profondément transformés (" dégradés ") et sont devenus par la suite des secteurs de conflits aigus entre éleveurs, d'un côté, et forestiers voulant reconstituer un peuplement "forestier », de l'autre.

D'autre part, la société a considéré jusqu'au $\mathrm{XIX}^{\bullet}$ siècle la forêt comme une source de bois inépuisable et surtout d'accès libre. Toute l'économie était fondée sur le bois et les besoins étaient considérables : bois de feu, de construction, d'artisanat, alimentation des cités proches et des industries locales. La misère générale n'a fait qu'accentuer la surexploitation.

L'action des forestiers a modifié tardivement ce tableau à partir de la Réformation de Froidour et par endroits seulement après la promulgation du Code forestier de 1827; elle reste donc plus facilement discernable grâce aux archives ou même sur le terrain. Elle s'est principalement exercée sur les massifs les mieux conservés au moment de la Réformation du XVII' siècle, c'est-à-dire les plus éloignés des villages ou les moins accessibles.

La mise au point de la théorie des étages bio-climatiques s'est fondée en particulier sur l'étude de la végétation dans les Pyrénées. Mais, même chez H. Gaussen qui fut un des premiers à faire des recherches dans les archives, le facteur humain fut largement sous-estimé. Beaucoup l'ont tout simplement escamoté. Il est d'ailleurs paradoxal de voir, au $\mathrm{XIX}^{\circ}$ siècle, les mêmes naturalistes qui s'horrifient des dégradations commises par les montagnards jeter les bases du concept de climax à partir de l'étude de ces végétations dégradées. L'organisation de la forêt est à réinterpréter, et les recherches historiques ou palynologiques récentes le permettent; il apparaît que l'étagement actuel peut en grande partie être considéré comme un étagement de production.

\section{La forêt de proximité}

Sur les bas de versants et les soulanes se trouve la chênaie dite collinéenne, à base de Chêne pubescent, pédonculé ou sessile (en fait, dans les Pyrénées Centrales, ces espèces sont presque toujours hybridées entre elles). Se mélangent aux Chênes : les Bouleaux, Ormes, 
Erables, Hêtres, Frênes, Merisiers, etc. Tous ces peuplements se rencontrent généralement dans des situations sèches et lumineuses : versants bien drainés ou calcaires, zone d'altitude inférieure à $1300 \mathrm{~m}$, exposition au sud ou à l'est, ce qui a conduit à en donner une interprétation exclusivement bioclimatique.

Dans le détail on s'aperçoit cependant que ces zones sont fondamentales pour l'économie villageoise : elles sont proches et facilement accessibles pour les besoins immédiats (bois, pâturage); bien exposées et déneigeant rapidement, elles sont parcourues à l'automne, au printemps et chaque fois que possible en hiver; enfin l'incendie est facile et fréquent sur ces versants secs, y maintenant des sous-bois clairs. De tels peuplements sont constitués par les espèces qui résistent le mieux aux mauvais traitements ou se régénèrent le plus vite, végétativement ou par semis, c'est-à-dire les espèces de lumière et de sols secs en général et les Chênes en particulier. Il est d'ailleurs significatif de voir le Chêne subsister seul dans les boisements à l'histoire la plus tourmentée.

La forêt de proximité est basse, claire, et les arbres tortueux, petits, en touffes y dominent; elle s'enfriche très vite de nos jours, et son aspect actuel, pourtant assez misérable, est souvent très différent de celui qu'elle avait voici un siècle : les photos montrent partout des peuplements encore plus rabougris et clairsemés. Il ne faut pas oublier que ces versants étaient parcourus par des centaines de bovins, ovins, caprins (qui affectionnaient les secteurs calcaires), des porcs et des animaux de bât, et qu'ils étaient brûlés le plus souvent possible. Bien souvent, la Chênaie collinéenne était même, voilà quelques décennies, une prairie, une culture ou un pâturage brouté à ras. La Chênaie de proximité s'individualise donc comme un peuplement de transition; ses limites correspondent fréquemment aux limites foncières: privé-communal, communal non soumis au régime forestier-communal soumis, communal-domanial.

Bien entendu les hommes n'ont pas inventé le Chêne. Les conditions de répartition de l'arbre sont essentiellement bio-climatiques, même si l'aire potentielle a pu être étendue par l'assèchement des milieux. Par contre, la Chênaie actuelle doit être considérée comme une création anthropique. Il y existe des exemples spectaculaires, sur des soulanes très exploitées depuis probablement fort longtemps (notamment à Melles, en Haute-Garonne), de Chênaies "sautant " tous les étages bioclimatiques pour finir à plus de $1800 \mathrm{~m}$ en garrigue subalpine, et qui commencent aujourd'hui à être recolonisées par le Hêtre et le Sapin (photo 1). Un cas semblable se retrouve dans les Montagnes Cantabriques où, dans les secteurs où le Hêtre a disparu, le Chêne Tauzin le remplace jusqu'à l'équivalent du subalpin (G. Bertrand). La variabilité génétique évidente du "Chêne Tauzin " dans les Montagnes Cantabriques est à mettre en parallèle avec celle, non moindre, du "Chêne sessile " dans les Pyrénées centrales. 


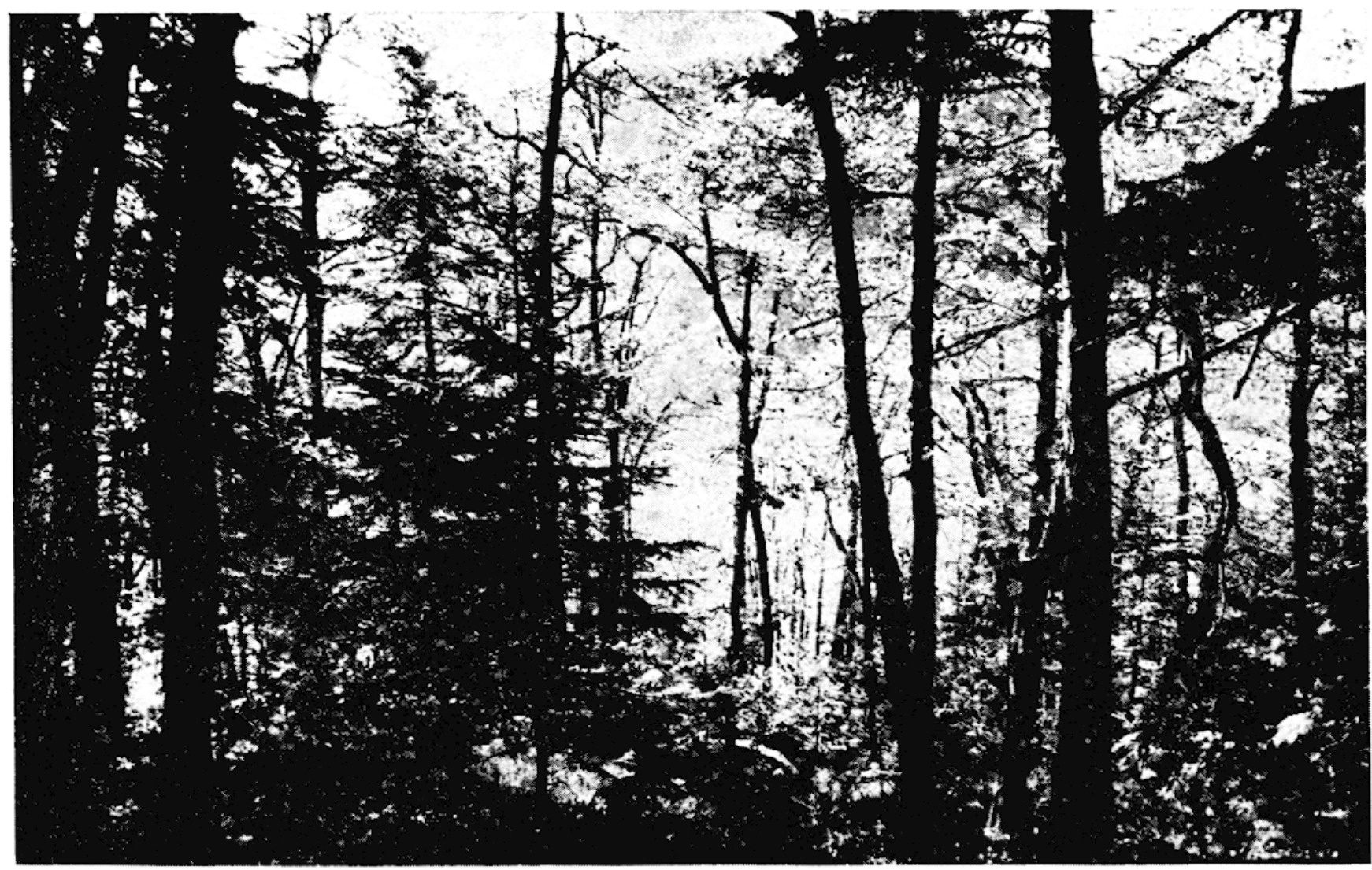

Pното 1

Vieille chênaie colonisée par le Sapin, dans la vallée de Melles

\section{La forêt de réserve}

Si les boisements de proximité servaient toute l'année pour tous les usages possibles, la Hêtraie avait une fonction moins variée, destinée surtout à la production de bois d'énergie et domestique. Dans ces forêts généralement plus éloignées des villages, l'exploitation individuelle faisait place à une exploitation organisée, collective ou commerciale : charbon, bois de boulange, bois de feu; la Hêtraie était utilisée comme une réserve de trésorerie par les montagnards. Ce type de gestion favorisait le taillis bas, de guère plus de $3 \mathrm{~m}$ de hauteur, où abondaient les jeunes brins aisément façonnables et transportables. D'autres formes, parfois spectaculaires, ont été induites par cette exploitation : la forêt de Hêtres têtards en est un exemple, d'ailleurs associée étroitement à une utilisation pastorale : sous les grosses souches espacées se développait une strate herbacée dense, entretenue par le feu. La coupe, le pâturage et l'incendie ont rapidement éliminé le Sapin, sensible à tous ces traitements et moins utile aux montagnards que le Hêtre (photo $n^{\circ} 2$ ). 


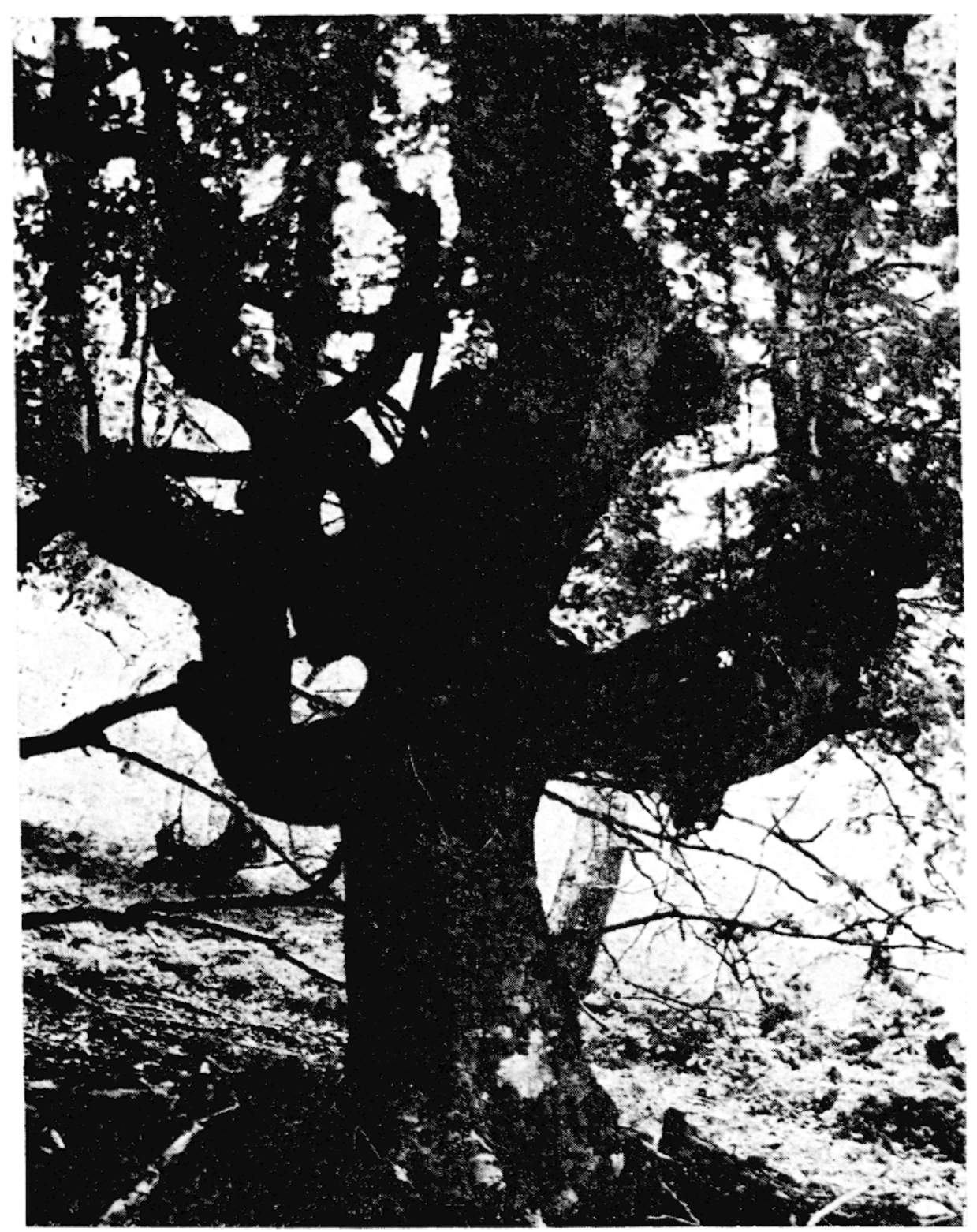

Pното 2

Hêtre têtard

Près des villages, là où les conditions climatiques étaient trop sévères ou sur les ombrées, la Hêtraie a également servi de forêt de proximité, donnant des formations de taillis extrêmement rabougris, parfois remplacés par des taillis de Bouleaux.

\section{La forêt forestière}

Il s'agit des Sapinières et Hêtraies-Sapinières de belle venue, gérées par les forestiers. Elles furent à l'origine protégées par leur éloigne- 
ment ou les difficultés d'accès, qui en gênaient l'exploitation, ainsi que part leur situation fréquente en ombrée qui leur donnait une médiocre valeur pastorale. Puis les forestiers royaux, et occasionnellement les propriétaires seigneuriaux, veillèrent à leur conservation. I_e Sapin a toujours été rigoureusement défendu et sélectionné au détriment des autres essences, sauf là où le transport était impossible et les dérivés du Hêtre plus avantageux, en Couserans, par exemple. Le cantonnement des droits d'usage dans un grand nombre de communes au XIX siècle a contribué à accentuer la séparation entre «belles forêts " protégées et "forêts paysannes" rabougries. On peut donc voir aujourd'hui voisiner, dans un environnement assez homogène, des peuplements purs de Sapins, Hêtres, voire Chênes. Ces séparations tendent aujourd'hui à disparaître. Le Sapin, délivré par l'exode rural de contraintes qui pesaient sur lui, commence désormais à recoloniser pratiquement tout l'espace montagnard. On le trouve aussi bien comme espèce pionnière des lisières supérieures vers $1800-1900 \mathrm{~m}$ que dispersé dans les taillis des collines de la bordure pyrénéenne vers $400 \mathrm{~m}$. Son expansion ne paraît limitée que par l'éloignement des porte-graines et par la trop grande sécheresse des versants les plus anthropisés.

Tout cela n'empêche pas l'existence de Sapinières pastorales, là où le rapport de force est resté longtemps en faveur des éleveurs et quand les conditions naturelles favorisent nettement le Sapin. Leur aspect est bien différent de celui des Sapinières forestières : arbres espacés, vieux, laissant se développer une strate herbacée dense, aux formes fourchues (produites par le broutage ou la coupe des jeunes sapins) on en candélabre (chablis facilités par l'espacement).

\section{Les écotones anthropiques}

Dans le détail des peuplements, en particulier sur les soulanes, on découvre souvent une répartition des essences qui exprime bien le rôle sélectif de la pression anthropique, par action volontaire ou involontaire; le phénomène est le plus net pour les écotones (1).

Le Chêne est fréquemment situé en lisière de Hêtraie, non pas seulement en étage inférieur mais, dans le cas de Hêtraies isolées au milieu des pâturages, en auréole. Le Hêtre se place de la même façon par rapport au Sapin. Il faut voir dans cette répartition le résultat des pressions très fortes et variées qui s'exercent sur les marges : broutage, piétinement, feu, etc. Les essences les plus résistantes et surtout celles qui se reproduisent bien végétativement sont ainsi favorisées. D'autres facteurs viennent se surajouter : héliophilie, résistance à la sécheresse de l'air.

1. Ecotone: désigne une zone de contact entre deux formations végétales. 
Mais on peut trouver des inversions d'écotones, principalement le Hêtre venant se placer en lisière de Chênaie. Il s'agit là d'une sélection volontaire du Hêtre dans les secteurs où il est peu abondant, le bois du Hêtre étant très apprécié pour l'artisanat. Par endroit, le Hêtre est tout simplement planté et constitue des haies facilement reconnaissables au contact de la forêt. Les plantations se retrouvent en bordure des propriétés privées, tandis que la sélection des peuplements s'opère dans les communaux.

On peut en définitive estimer que la forêt "climax", telles qu'elle pourrait exister dans les conditions bio-climatiques actuelles des Pyrénées, serait un mélange beaucoup plus confus que l'étagement classique. Evidemment les proportions respectives des essences varieraient selon l'exposition, l'altitude, le substrat; il n'y a pas là à contester quoi que ce soit. Mais les étages bien tranchés que l'on peut observer de nos jours représentent davantage un compromis entre les conditions naturelles et la pression de la société montagnarde. Dans chaque lieu donné ce compromis a favorisé les espèces en place les plus rustiques et résistantes (avec une exception : le Châtaignier, introduit). De ce point de vue, le Chêne est bien l'arbre caractéristique de la forêt paysanne, le Chêne étant pris au sens large, la souplesse de comportement des espèces n'ayant d'égale que leur souplesse génétique.

Il est significatif à ce propos que sur la carte de la végétation de Foix dressée par H. Gaussen la Chênaie soit systématiquement sousestimée : les facteurs de sa répartition ne découlent pas d'un modèle bio-climatique exclusif. 


\section{Bibliographie}

L. BAUdin, La forme des arbres dans les forêts pyrénéennes La forêt de Guzet (Ariège). Mémoire de DEA, 45 p., Institut de géographie Université de Toulouse-Le Mirail, Toulouse, 1983.

C. FRUHAUf, Forêt et société. De la forêt paysanne à la forêt capitaliste en pays de Sault sous l'Ancien Régime (1670-1791). Toulouse, 1980, CNRS, 302 p.

G. Jalut, A. A. Esteban, Ph. Pages, M. Mardones, Quelques aspects de l'action de I'homme sur le milieu montagnard pyrénéen: conséquences phytogéographiques. Toulouse, 1982, Colloque international "Ecologie et biogéographie des milieux montagnards et de haute altitude ${ }^{\text {, Gabas. }}$

J.-P. MÉraIlik, Le feu pastoral dans les Pyrénées centrales (Barousse, Oueil, Larboust). Toulouse, 1981, CNRS, 294 p.

- Forêts, pâturages et société montagnarde: 1. La soulane d'Oueil. 20 p., 1 carte $1 / 25000$. 2. La soulane de Melles. 15 p., 1 carte 1/25 000 . (à paraître).

RÉsumÉ. - L'étagement de la végétation dans les Pyrénées centrales correspond en grande partie à un étagement de production. L'intensité et la durée de l'action humaine a sélectionné les principales espèces forestières selon leur adaptation aux types d'usage des espaces considérés. Les chênes ( $Q$. pubescens, pedunculata et sessiliflora) apparaissent comme les espèces les plus anthropophiles.

Summary. - The farmer-exploited forest in the Pyrénées centrales. The natural terracing of the vegetation in the Pyrénées centrales to a large extent corresponds to a terracing of exploitation. The strenuous labour of Man has selected the main tree species according to their fitness to the use of their environment. Oaks (Quercus pubescens, pedunculata and sessiliflora) appear to be the most anthropophilic species.

Resumen. - Los bosques « Rurales » del Pirineo central. El escalonamiento de la vegetación del Pirineo central corresponde en parte a un escalonamiento productivo. La intensidad y la duración de la intervención humana han seleccionado las principales especies forestales según su adaptación a los tipos de aprovechamiento en determinados espacios. Las especies más antropófilas parecen ser el Quercus pubescens, el Quercus pedunculata (carvallo), el Quercus sessiliflora (roble albar).

Mors-Clés. - Pyrénées centrales, forêt, écotones, étagement de la végétation, dendromorphologie, chêne, production forestière, époque contemporaine. 